\title{
Estudio sobre compromiso y expectativas de autoeficacia académica en estudiantes universitarios de grado
}

\author{
Pedro Ricardo Álvarez-Pérez \\ David López-Aguilar \\ Yaritza Garcés-Delgado \\ Universidad de La Laguna. España. \\ palvarez@ull.edu.es \\ dlopez@ull.edu.es \\ ygarcesd@ull.edu.es
}

Recibido: $13 / 10 / 2020$

Aceptado: 12/3/2021

Publicado: 5/7/2021

\section{Resumen}

El análisis de las expectativas académicas y del nivel de compromiso hacia el grado que cursa el alumnado universitario permite conocer hasta qué punto es capaz de realizar una autoevaluación, generalmente subjetiva, sobre la probabilidad de lograr buenos resultados en sus estudios. La finalidad de esta investigación es analizar la incidencia que el compromiso académico tiene en las creencias de autoeficacia y en las expectativas de rendimiento en universitarios de grado. La muestra la constituyen 502 alumnos de primer y segundo curso de los grados ofertados por la Facultad de Educación de la ULL. Se administró la escala validada de compromiso académico Utrech Work Engagement Scale-UWES-17 y, con el fin de valorar las expectativas de rendimiento académico, se preguntó qué calificaciones esperaban obtener al final del primer semestre. Los resultados revelaron que los estudiantes que tenían creencias favorables hacia el logro de buenas calificaciones eran quienes obtenían mejores puntajes en la escala de compromiso académico. La incidencia que ejerce dicho parámetro en las expectativas de resultados del alumnado universitario abre una línea de actuación importante en la educación superior, encaminada a lograr un nivel más elevado de adaptación, persistencia y éxito en los estudios.

Palabras clave: compromiso académico; creencias de autoeficacia; expectativas de resultados; educación universitaria

Resum. Estudi sobre compromis i expectatives d'autoeficàcia acadèmica en estudiants universitaris de grau

L'anàlisi de les expectatives acadèmiques i del nivell de compromís vers el grau que cursa l'alumnat universitari permet conèixer fins a quin punt és capaç de realitzar una autoavaluació, generalment subjectiva, sobre la probabilitat d'aconseguir bons resultats en els seus estudis. La finalitat d'aquesta investigació és analitzar la incidència que el compromís acadèmic té en les creences d'autoeficàcia i en les expectatives de rendiment en universitaris de grau. La mostra la constitueixen 502 alumnes de primer i segon curs dels graus oferts per la Facultat d'Educació de la ULL, a qui se'ls va administrar l'escala validada de 
compromís acadèmic Utrech Work Engagement Scale-UWES-17 i, per tal de valorar les expectatives de rendiment acadèmic, se'ls va preguntar quines qualificacions esperaven obtenir a la fi del primer semestre. Els resultats van revelar que els estudiants que tenien creences favorables cap a l'assoliment de bones qualificacions eren els que obtenien millors puntuacions en l'escala de compromís acadèmic. La incidència que exerceix aquest paràmetre en les expectatives de resultats de l'alumnat universitari obre una línia d'actuació important en l'educació superior, encaminada a aconseguir un nivell més elevat d'adaptació, persistència i èxit en els estudis.

Paraules clau: compromís acadèmic; creences d'autoeficàcia; expectatives de resultats; educació universitària

Abstract. Study on Commitment and Expectations of Academic Self-Efficacy in Undergraduate Students

The analysis of academic expectations and level of commitment to the study program allows us to determine to what extent university students are capable of carrying out generally subjective self-assessments of the probability of achieving good academic outcomes. The purpose of this study is to analyze the incidence that academic commitment has on self-efficacy beliefs and performance expectations of undergraduate university students. The sample consisted of 502 students who were in the first and second year of bachelor's degree programs at the Faculty of Education of the University of La Laguna, Spain. The validated Utrecht Work Engagement Scale (UWES-17) was administered to the students to measure their academic commitment. To assess their expectations for academic performance, the students were asked what grades they expected to obtain at the end of the first semester. The results revealed that students who had favorable beliefs regarding the achievement of good grades in the subjects obtained better scores on the academic engagement scale. The incidence that academic commitment has on the expectations of outcomes of university students opens an important line of action in higher education aimed at achieving better adaptation, perseverance and academic success.

Keywords: academic commitment; academic self-efficacy; expectations of results; higher education

\author{
Sumario \\ 1. Introducción 4. Conclusiones \\ 2. Material y método Referencias bibliográficas
}

3. Resultados

\title{
1. Introducción
}

La educación superior ha experimentado en la última década cambios importantes que han situado al estudiante en el primer plano del proceso de enseñanza y aprendizaje. La transmisión de saberes por parte del profesorado ha dejado paso a un modelo en el que se pide al alumnado que sea el protagonista y el gestor de su adquisición de conocimientos. Al mismo tiempo se le reclama que sea activo, reflexivo, autónomo, estratégico, que entienda lo que aprende 
y que aprenda de forma permanente. Este cambio de orientación en el enfoque de la formación ha tropezado con la dificultad que para muchos estudiantes supone adaptarse a este nuevo modelo y gestionar su educación de manera autónoma y eficiente.

Muchos alumnos tienen diariamente que hacer frente a una diversidad de actividades de aprendizaje y afrontar cargas académicas prolongadas, lo que les conduce a situaciones de estrés, ansiedad, desmotivación y expectativas negativas hacia su formación (Belhumeur, Barrientos y Renata-Salazar, 2016). Estas dificultades para afrontar las tareas académicas derivan en muchos casos en fracaso o abandono de los estudios. El abandono académico es uno de los graves problemas de la educación superior, siendo el primer curso uno de los momentos más críticos donde se vienen detectando las mayores cifras de deserción (Figuera, Torrado, Dorio y Freixa, 2015). Desde hace tiempo se viene investigando sobre los factores que lo provocan y se han propuesto modelos explicativos para intervenir sobre el mismo (Tinto, 1975; Bethencourt et al., 2008; Rué, 2014; Bernardo, Cerezo, Rodríguez y Tuero, 2015). De estos estudios se concluye que el abandono es un problema multicausal y que son muchas las variables, de muy distinta naturaleza, que explican la permanencia o la desvinculación académica (Arriaga, Burillo, Carpeñol y Casaravilla, 2011; Chen, 2012). Los resultados del estudio de Íniguez, Elboj y Valero (2016) evidenciaron esta pluralidad de variables, ya que para los alumnos fueron factores extracurriculares, como la dificultad para compaginar estudios y trabajo, los que influyeron en el abandono y, para el profesorado, la escasa motivación o la falta de un perfil de acceso adecuado. Es por ello que, como señalan Torrado y Figuera (2019), la prevención del abandono académico en la educación superior se ha convertido en uno de los retos más importantes de la política universitaria actual. El análisis de factores vinculados al abandono, que puedan ayudar a comprender las causas que inciden en la adaptación inicial del alumnado y en la mejora de las cifras de retención, se apunta como una de las importantes líneas estratégicas a seguir.

De acuerdo con esta perspectiva, una de las variables que ha cobrado un gran interés en los últimos tiempos en la enseñanza es el compromiso académico, por la influencia que ejerce a la hora de conseguir una buena adaptación social y universitaria, contribuir a la permanencia, lograr un aprendizaje activo y alcanzar buenos resultados académicos (Schaufeli et al., 2002a). Se define como un estado de bienestar psicológico que integra tres factores principales: el vigor (la energía y la resistencia mental durante el tiempo en el que se realiza una tarea), la dedicación (motivación e implicación mostrada durante la realización activa de una tarea) y la absorción (hace referencia al estado de concentración profunda durante la realización del trabajo académico) (Martínez y Salanova, 2003; Schaufeli y Bakker, 2003). El compromiso se considera un estado estable (no algo puntual o circunstancial), y describe la implicación y la satisfacción del alumnado con su proceso de aprendizaje, así como la concentración y el esfuerzo por alcanzar las metas formativas (Coates y McCormick, 2014; Cavazos y Encinas, 2016). 
Como señalan Savage, Strom, Ebesu y Aune (2017), el compromiso académico es un factor de gran relevancia para entender la persistencia del alumnado en su proceso formativo. También en el análisis de Álvarez-Pérez, LópezAguilar y Valladares-Hernández (2021) se demostró que aquellos estudiantes que puntuaron más alto en una escala de compromiso académico eran los que nunca habían repetido curso en los años previos. Esto permite destacar el valor predictivo del compromiso sobre el rendimiento en los estudios, ya que, como se ha señalado en otras investigaciones (Parra, 2010; Martos et al., 2018), el alumnado implicado y comprometido realiza su trabajo con energía, está más concentrado, disfruta con su formación, está motivado y demuestra mayor confianza en sus posibilidades. Conseguir buenos resultados de aprendizaje exige mucho esfuerzo y dedicación y si los estudiantes no están preparados psicológicamente y no tienen un alto nivel de compromiso con lo que hacen, pueden verse superados y afectados sus planes académicos (Schauffeli y Salanova, 2007; Bresó y Gracia, 2007). Los estudiantes comprometidos con su formación tienen una alta implicación en el aprendizaje y muestran un buen desempeño en las labores académicas. Por eso y en oposición al compromiso, el burnout se refiere al síndrome de estar quemado, al desgaste profesional, a la dificultad de continuar una tarea, a la tendencia al abandono y a la falta de logro personal (Gil-Monte, 2003). Es el caso de muchos educandos que se ven superados por el estrés que genera el proceso de aprendizaje y terminan abandonando la formación.

El compromiso académico se relaciona con las creencias de autoeficacia estudiantil, de modo que los alumnos con un alto nivel de compromiso suelen autopercibirse como capaces para el aprendizaje y para alcanzar buenos resultados en los estudios (Casas y Blanco, 2017). Según Bandura (1982), las expectativas de autoeficacia dependen de la creencia de cada uno respecto a si posee las capacidades necesarias para lograr sus objetivos. En el plano académico, las creencias de autoeficacia son los pensamientos que tienen los estudiantes acerca de su capacidad y de su autorregulación para conseguir los objetivos formativos. Las creencias, motivaciones y expectativas que tiene el alumnado que inicia las clases universitarias, que se modulan en interacción con el contexto, con las exigencias del trabajo académico, con la interacción que mantienen con el profesorado o con el ambiente de aula, influyen de manera decisiva en la integración social y en la continuación o deserción de la formación (Wilkins, Butt, Kratochvil y Balakrishnan, 2016; Jurado, Figuera y Llanes, 2019). Desde el enfoque sociocognitivo del desarrollo de la carrera, se señala que las expectativas de autoeficacia académica tienen un efecto directo en la satisfacción y la permanencia en los estudios, lo cual constituye un freno al problema del abandono (Lent et al., 2017). También Tinto (2017) llama la atención sobre la importancia que tiene la autopercepción del alumnado acerca de sus capacidades sobre la adaptación y el sentido de pertenencia a la institución, lo cual es un buen predictor del logro de metas y de resultados formativos.

En referencia a estos factores que se relacionan estrechamente con el abandono de los estudios, el objetivo de esta investigación fue analizar, en univer- 
sitarios de grado, si su compromiso incidía en las expectativas y en las creencias de autoeficacia académica.

\section{Material y método}

\subsection{Hipótesis}

Para este trabajo se definió como hipótesis nula $\left(\mathrm{H}_{0}\right)$ que los universitarios de grado de la Facultad de Educación de la ULL con bajos niveles de compromiso e implicación académica tenían bajas expectativas de rendimiento en sus estudios. Por el contrario, como hipótesis alternativa $\left(\mathrm{H}_{1}\right)$, se planteó que el conjunto de universitarios de grado de la Facultad de Educación de la ULL con altos niveles de compromiso académico mostraba mayores expectativas de lograr un buen rendimiento en sus estudios.

\subsection{Participantes}

La población objeto de análisis estaba constituida por alumnado que seguía los cursos primero y segundo de grados de la Facultad de Educación de la ULL. Según datos oficiales de matriculación ofrecidos por este centro, en el momento de la administración de la prueba de recogida de datos estaban inscritos 2.216 estudiantes que reunían las características descritas. Asumiendo un error del $\pm 5 \%$ y una confianza del $95 \%$, participaron en la investigación 502 estudiantes, lo que permitió trabajar con un margen de error del $\pm 3,9 \%$ y una confianza del $95,3 \%$. Las características de la muestra definitiva se presentan en la tabla 1 .

Tabla 1. Características de la muestra

\begin{tabular}{ll}
\hline Edad & Mínimo: $17 ;$ máximo: $47 ; \bar{x}=19,66 ; s d=3,48$. \\
Sexo & Mujeres: $23,3 \%(n=117)$. \\
& Hombres: $76,7 \%(n=385)$. \\
& Pedagogía: $15,5 \%(n=78)$. \\
Titulación & Maestro en Educación Infantil: $28,5 \%(n=143)$. \\
& Maestro en Educación Primaria: $56,0 \%(n=281)$. \\
Curso & Primero: $75,95 \%(n=381)$. \\
& Segundo: $24,1 \%(n=121)$. \\
\hline
\end{tabular}

Fuente: elaboración propia.

\subsection{Instrumento de recogida de datos}

Para medir el nivel de compromiso académico se utilizó la escala Utrech Work Engagement Scale (Schaufeli, Salanova, González-Romá y Bakker, 2002b). Esta prueba contenía 17 ítems tipo Likert (0 indicaba la puntuación más baja, y 6, la más alta) distribuidos en tres variables: vigor, absorción y dedicación (tabla 2). De acuerdo con Carmona-Halty, Schaufeli y Salanova (2017), el 
Tabla 2. Escala de compromiso utilizada en el cuestionario

\begin{tabular}{|c|c|c|c|}
\hline Dimensión & Variables & Ítems & Cod. \\
\hline \multirow{17}{*}{$\begin{array}{l}\text { Compromiso } \\
\text { académico }\end{array}$} & \multirow{6}{*}{ Vigor } & $\begin{array}{l}\text { Cuando realizo tareas de clase me siento con fuerza y } \\
\text { lleno de energía. }\end{array}$ & $\mathrm{v} 1$ \\
\hline & & $\begin{array}{l}\text { Me siento con fuerza y animado cuando estoy haciendo } \\
\text { actividades de clase. }\end{array}$ & v2 \\
\hline & & $\begin{array}{l}\text { Cuando me levanto por la mañana me apetece ir a } \\
\text { clase, ponerme a estudiar o hacer tareas de clase. }\end{array}$ & v3 \\
\hline & & $\begin{array}{l}\text { Puedo estar estudiando de manera continuada durante } \\
\text { periodos largos de tiempo (sin que me resulte aburrido). }\end{array}$ & v4 \\
\hline & & $\begin{array}{l}\text { A pesar de las dificultes que encuentre, me considero } \\
\text { una persona constante y perseverante a la hora de } \\
\text { afrontar las tareas de clase. }\end{array}$ & v5 \\
\hline & & $\begin{array}{l}\text { Incluso si no me encuentro bien, no dejo de realizar mis } \\
\text { tareas de clase. }\end{array}$ & v6 \\
\hline & \multirow{5}{*}{ Dedicación } & $\begin{array}{l}\text { Creo que los estudios que estoy realizando tienen sen- } \\
\text { tido para mí. }\end{array}$ & d1 \\
\hline & & $\begin{array}{l}\text { Estoy entusiasmado con los estudios universitarios que } \\
\text { estoy realizando. }\end{array}$ & d2 \\
\hline & & $\begin{array}{l}\text { Los estudios que estoy cursando me motivan a hacer } \\
\text { cosas nuevas relacionadas con la formación que estoy } \\
\text { realizando. }\end{array}$ & d3 \\
\hline & & $\begin{array}{l}\text { Estoy orgulloso de estar cursando esta titulación univer- } \\
\text { sitaria. }\end{array}$ & $\mathrm{d} 4$ \\
\hline & & $\begin{array}{l}\text { Cursar estos estudios universitarios es un reto importan- } \\
\text { te para mí. }\end{array}$ & d5 \\
\hline & \multirow{6}{*}{ Absorción } & $\begin{array}{l}\text { Se me pasa el tiempo volando cuando realizo las tareas } \\
\text { de clase. }\end{array}$ & a1 \\
\hline & & $\begin{array}{l}\text { Me olvido de todo lo que pasa a mi alrededor cuando } \\
\text { estoy concentrado en mis estudios. }\end{array}$ & a2 \\
\hline & & $\begin{array}{l}\text { Me siento feliz cuando estoy realizando las tareas de } \\
\text { clase. }\end{array}$ & a3 \\
\hline & & $\begin{array}{l}\text { Estoy implicado e inmerso en los estudios que estoy } \\
\text { cursando. }\end{array}$ & a4 \\
\hline & & $\begin{array}{l}\text { Cuando realizo las tareas de clase estoy tan implicado } \\
\text { que pierdo la noción del tiempo. }\end{array}$ & a5 \\
\hline & & Me resulta difícil dejar de realizar las tareas de clase. & a6 \\
\hline
\end{tabular}

Fuente: elaboración propia.

vigor hace referencia a la fuerza y a la energía que una persona muestra durante la realización de una tarea, incluso a pesar de las dificultades. Por su parte, la absorción es la capacidad de estar implicado y concentrado profundamente en una determinada actividad, lo que lleva a una falta de noción del tiempo. Finalmente, la dedicación se refiere a la alta motivación, al interés y a la participación activa en la ejecución de una tarea. Los valores de fiabilidad obtenidos por 
Schaufeli et al. (2002b) en la Utrech Work Engagement Scale mediante el cálculo del coeficiente alfa de Cronbach $(\alpha)$ superaron los valores críticos propuestos por la literatura. Asimismo, estos autores ensayaron un modelo de ecuaciones estructurales para confirmar la constitución subyacente de esta escala. Los índices de ajuste del modelo ensayado (RMSEA, GFI, AGFI, NFI, NNFI, TLI, CFI) respondieron adecuadamente a los valores establecidos.

Además de esta escala, para valorar las expectativas de rendimiento académico, se incluyó una pregunta en la que se consultó la calificación que esperaban obtener tras la evaluación del primer semestre. Por último, se añadieron ítems relacionados con los datos sociodemográficos.

\subsection{Procedimiento, análisis e interpretación de los datos}

El proceso de recogida de datos tuvo lugar en el curso 2019-2020 y consistió en la aplicación presencial del cuestionario diseñado. Para ello se contactó con docentes de los dos primeros cursos de los grados de la Facultad de Educación, para que permitieran administrar la prueba. En el momento de aplicarla se ofreció a los participantes un consentimiento informado, en el que se indicó las finalidades de la investigación, las garantías del anonimato y las instrucciones para responder.

Finalizada la recogida de información, se creó una base de datos con el programa Microsoft Excel, que fue almacenada en formato CSV para ser tratada posteriormente con el software R-Studio (versión 1.2.5001). Este apoyo informático permitió identificar los posibles datos perdidos, eliminar los casos atípicos multivariantes, realizar análisis de tendencia central, revisar la distribución de la normalidad de los datos, valorar la consistencia interna y la fiabilidad de la escala utilizada y realizar análisis de contraste. Además, para identificar el tamaño del efecto de las diferencias encontradas, se empleó el coeficiente de épsilon al cuadrado $\left(E_{R}^{2}\right)$, el cual fue calculado en el programa Excel con la siguiente expresión matemática, donde $H$ hacía referencia a la puntuación obtenida en la prueba de Kruskal-Wallis, y $n$, al número de observaciones realizadas:

$$
E_{R}^{2}=\frac{H}{\left(n^{2}-1\right) /(n+1)}
$$

Los resultados obtenidos con este coeficiente fueron interpretados a partir de los valores propuestos por Faul, Erdfelder, Lang y Buchner (2007) y Tomczak y Tomczak (2014). 


\section{Resultados}

\subsection{Depuración de datos y análisis descriptivos preliminares}

Como punto de partida se comprobó que la base de datos no contuviera errores que distorsionaran los exámenes estadísticos. Para ello se revisó que el rango de respuestas de los ítems propuestos estuviera en el margen establecido. En este caso, se confirmó que la información imputada en la base de datos referidos a la escala tipo Likert estuviera entre los valores 0 y 6 . Asimismo, se revisó que no existieran casos perdidos.

También se comprobó que la base de datos no contuviera datos atípicos multivariantes (outliers). Para ello se calculó la distancia de Mahalanobis, la cual permite identificar si un participante está superando el punto de corte establecido a partir del que se sitúan los casos extremos (Muñoz y Amón, 2013). Asumiendo un valor $p=0,95$, el resultado de la distancia de Mahalanobis fue de 27,58. Este valor crítico determinó la necesidad de eliminar 75 casos, con lo que la muestra definitiva se situó en un total de 427 estudiantes. En la tabla 3 se presenta la distribución de las puntuaciones de tendencia central obtenidas en la escala de medida cumplimentada por la muestra definitiva.

Tabla 3. Datos descriptivos de la escala de compromiso académico empleada

\begin{tabular}{|c|c|c|c|c|c|}
\hline & $n$ & $\overline{\mathbf{x}}$ & sd & M & $M_{e}$ \\
\hline $\mathrm{v} 1$ & \multirow{17}{*}{427} & 3.597 & 1.401 & 4 & 4 \\
\hline v2 & & 3.646 & 1.378 & 4 & 4 \\
\hline v3 & & 3.028 & 1.628 & 4 & 3 \\
\hline v4 & & 3.333 & 1.614 & 4 & 4 \\
\hline v5 & & 4.419 & 1.461 & 5 & 5 \\
\hline v6 & & 3.623 & 1.807 & 5 & 4 \\
\hline d1 & & 4.946 & 1.335 & 6 & 5 \\
\hline d2 & & 4.761 & 1.494 & 6 & 5 \\
\hline d3 & & 4.358 & 1.472 & 5 & 5 \\
\hline $\mathrm{d} 4$ & & 5.115 & 1.393 & 6 & 6 \\
\hline d5 & & 4.845 & 1.388 & 6 & 5 \\
\hline a1 & & 3.492 & 1.487 & 3 & 4 \\
\hline a2 & & 3.478 & 1.531 & 4 & 4 \\
\hline a3 & & 3.253 & 1.495 & 4 & 3 \\
\hline $\mathrm{a} 4$ & & 4.557 & 1.416 & 5 & 5 \\
\hline a5 & & 3.417 & 1.608 & 4 & 4 \\
\hline a6 & & 3.585 & 1.770 & 5 & 4 \\
\hline
\end{tabular}

Fuente: elaboración propia. 
El análisis de la multicolineidad permitió valorar la posible redundancia de los ítems integrados en la escala de compromiso académico. Para ello se realizó una correlación bivariada y se comprobó que las puntuaciones no superaran los valores críticos propuestos por Holgado, Suárez y Morata (2019): $r \leq 0,85$.

Con el objetivo de someter a prueba la hipótesis, se comprobó la distribución normal de los datos para identificar las pruebas de contrastes a realizar. En concreto, se analizó la normalidad a partir de la revisión de los valores de la asimetría, la curtosis, el test de Shaphiro Wilks y el de Kolmogorov Smirnov. Para su interpretación se tuvieron en cuenta las aportaciones de George y Mallery (2001). De acuerdo con la información presentada en la tabla 4, los datos confirmaron que no seguían una distribución normal, por lo que las pruebas de contraste realizadas fueron no paramétricas.

Tabla 4. Análisis de la normalidad

\begin{tabular}{|c|c|c|c|c|c|}
\hline \multirow[t]{2}{*}{ Ítems } & \multicolumn{2}{|c|}{ Skewness (asimetría) } & \multirow{2}{*}{$\begin{array}{l}\text { Kurtosis } \\
\text { (curtosis) }\end{array}$} & \multirow{2}{*}{$\begin{array}{l}\text { Shaphiro Wilks } \\
\quad(p \text { valor })\end{array}$} & \multirow{2}{*}{$\begin{array}{l}\text { Kolmogorov } \\
\text { Smirnov KS } \\
\text { ( } p \text { valor) }\end{array}$} \\
\hline & Skewness & $p$ & & & \\
\hline v1 & $-0,238$ & 0,043 & $-0,579$ & & \\
\hline v2 & $-0,456$ & $<0,001$ & $-0,317$ & & \\
\hline v3 & $-0,195$ & 0,096 & $-0,901$ & & \\
\hline v4 & $-0,250$ & 0,034 & $-0,795$ & & \\
\hline v5 & $-0,800$ & $<0,001$ & $-0,200$ & & \\
\hline v6 & $-0,309$ & $<0,001$ & $-1,056$ & & \\
\hline d1 & $-1,417$ & $<0,001$ & 1,280 & & \\
\hline d2 & $-1,255$ & $<0,001$ & 0,801 & & \\
\hline d3 & $-0,875$ & $<0,001$ & 0,062 & $<0,001$ & $<0,001$ \\
\hline $\mathrm{d} 4$ & $-1,622$ & $<0,001$ & 1,599 & & \\
\hline d5 & $-1,090$ & $<0,001$ & 0,182 & & \\
\hline a1 & $-0,331$ & 0,005 & $-0,434$ & & \\
\hline a2 & $-0,268$ & 0,023 & $-0,922$ & & \\
\hline a3 & $-0,285$ & 0,016 & $-0,621$ & & \\
\hline a4 & $-0,946$ & $<0,001$ & 0,101 & & \\
\hline a5 & $-0,383$ & 0,001 & $-0,708$ & & \\
\hline a6 & $-0,296$ & 0,012 & $-0,964$ & & \\
\hline
\end{tabular}

Fuente: elaboración propia.

Finalmente, se calculó el índice de fiabilidad de la escala de medida. Al cumplir la escala con los supuestos de tau-equivalencia, unidimensionalidad y continuidad de medida (Cho, 2016; Raykov y Marcoulides, 2017), se utilizó el coeficiente alfa de Cronbach. Los datos generales y específicos (tabla 5) 
Tabla 5. Análisis de fiabilidad

\begin{tabular}{|c|c|c|c|c|}
\hline Variables & Ítems & \multicolumn{3}{|c|}{ Alfa de Cronbach $(\alpha)$} \\
\hline \multirow{6}{*}{ Vigor } & $\mathrm{v} 1$ & 0,86 & \multirow{6}{*}{0,89} & \multirow{17}{*}{0,96} \\
\hline & v2 & 0,86 & & \\
\hline & v3 & 0,86 & & \\
\hline & v4 & 0,86 & & \\
\hline & v5 & 0,87 & & \\
\hline & v6 & 0,89 & & \\
\hline \multirow{5}{*}{ Dedicación } & d1 1 & 0,92 & \multirow{5}{*}{0,94} & \\
\hline & d2 & 0,91 & & \\
\hline & d3 & 0,94 & & \\
\hline & $\mathrm{d} 4$ & 0,91 & & \\
\hline & d5 & 0,93 & & \\
\hline \multirow{6}{*}{ Absorción } & a1 & 0,88 & \multirow{6}{*}{0,90} & \\
\hline & a2 & 0,88 & & \\
\hline & a3 & 0,87 & & \\
\hline & a4 & 0,87 & & \\
\hline & a5 & 0,87 & & \\
\hline & a6 & 0,90 & & \\
\hline
\end{tabular}

Fuente: elaboración propia.

que arrojó este índice demostraron que la escala de medida utilizada disponía de una buena consistencia interna (Ventura-León y Caycho-Rodríguez, 2017).

\subsection{Análisis de contraste y tamaño del efecto}

Los resultados generales del análisis de contraste realizado mediante la prueba $\mathrm{H}$ de Kruskal-Wallis manifestaron que el grupo de estudiantes que esperaba obtener calificaciones altas era el de los que mayor compromiso tenían con los estudios $(H=53.577 ; p<0.001)$ (tabla 6). El valor obtenido en el coeficiente épsilon al cuadrado $\left(E_{R}^{2}\right)$ ) demostró que el tamaño del efecto de las diferencias encontradas fue bajo $\left.\left(E_{R}^{2}\right)=0,125\right)$.

De manera más específica, se detectaron diferencias estadísticamente significativas $(p \leq 0,05)$ en las distintas variables que integran la escala de compromiso académico (tabla 7). Los estudiantes que esperaban alcanzar calificaciones de sobresaliente eran los que mayor puntuación obtuvieron en las variables vigor $\left(H=54.672 ; p>0,001 ; E_{R}^{2}=0,128\right)$, dedicación $(H=33.270$; $\left.p>0,001 ; E_{R}^{2}=0,078\right)$ y absorción $\left(H=50.497 ; p>0,001 ; E_{R}^{2}=0,118\right)$. Siguiendo los valores críticos propuestos por Tomczak y Tomczak (2014) y Faul et al. (2007) para la interpretación del tamaño del efecto, las diferencias encontradas se situaron en una magnitud media $\left(E_{R}^{2} \geq 0,06\right)$. 
Tabla 6. Análisis de contraste de la puntuación general obtenida en la escala de compromiso académico

\begin{tabular}{lrrrrc}
\hline Muestras & $\boldsymbol{n}$ & Rangos & $\boldsymbol{H}$ & $\boldsymbol{p}$ & $\begin{array}{c}\text { Coeficiente épsilon } \\
\text { al cuadrado }\left(\boldsymbol{E}_{\boldsymbol{R}}^{2}\right)\end{array}$ \\
\hline Aprobados & 136 & 152,25 & & & \\
\hline Notables & 264 & 240,20 & & \\
\hline Sobresalientes & 25 & 279,06 & 53.577 & $<0,001$ & 0,125 \\
\hline Matrículas de honor & 2 & 141,75 & & & \\
\hline
\end{tabular}

Fuente: elaboración propia.

Tabla 7. Análisis de contraste de las variables de la escala de compromiso académico

\begin{tabular}{|c|c|c|c|c|c|c|}
\hline Variables & Muestras & $n$ & Rangos & $H$ & $p$ & $\begin{array}{l}\text { Coeficiente épsilon } \\
\text { al cuadrado }\left(E_{R}^{2}\right)\end{array}$ \\
\hline \multirow{4}{*}{ Vigor } & Aprobados & 136 & 153,37 & \multirow{4}{*}{54.672} & \multirow{12}{*}{$<0,001$} & \multirow{4}{*}{0,128} \\
\hline & Notables & 264 & 239,10 & & & \\
\hline & Sobresalientes & 25 & 288,12 & & & \\
\hline & Matrículas de honor & 2 & 97,00 & & & \\
\hline \multirow{4}{*}{ Dedicación } & Aprobados & 136 & 164,81 & \multirow{4}{*}{33.270} & & \multirow{4}{*}{0,078} \\
\hline & Notables & 264 & 235,65 & & & \\
\hline & Sobresalientes & 25 & 256,56 & & & \\
\hline & Matrículas de honor & 2 & 169,50 & & & \\
\hline \multirow{4}{*}{ Absorción } & Aprobados & 136 & 154,05 & \multirow{4}{*}{50.497} & & \multirow{4}{*}{0,118} \\
\hline & Notables & 264 & 239,06 & & & \\
\hline & Sobresalientes & 25 & 279,60 & & & \\
\hline & Matrículas de honor & 2 & 162,50 & & & \\
\hline
\end{tabular}

Fuente: elaboración propia.

Habiendo aplicado la prueba $\mathrm{H}$ de Kruskal-Wallis se encontraron diferencias significativas en las puntuaciones obtenidas en los distintos ítems de la escala de compromiso (tabla 8). De manera más específica, las diferencias detectadas señalaron que los alumnos con altas expectativas de rendimiento en los estudios eran los que mayores puntuaciones obtuvieron en la totalidad de ítems de la escala. En la variable vigor, este grupo de estudiantes se caracterizaba por realizar las tareas de clase con fuerza y energía $(H=27.729 ; p>0,001$; $\left.E_{R}^{2}=0,065\right)$, por levantarse por las mañanas con ganas e ilusión de ir a clase $\left(H=34.188 ; p>0,001 ; E_{R}^{2}=0,080\right)$, por seguir perseverando a pesar de las dificultades que encontraban en el estudio $\left(H=49.415 ; p>0,001 ; E_{R}^{2}=0,115\right)$ y por sentirse bien cuando continuaban con la realización de sus tareas académicas $\left(H=37,57 ; p>0,001 ; E_{R}^{2}=0,088\right)$. Además, y relacionado con la dedicación, el conjunto de estudiantes que manifestó tener altas expectativas 
Tabla 8. Análisis de contraste de los ítems de la escala de compromiso académico

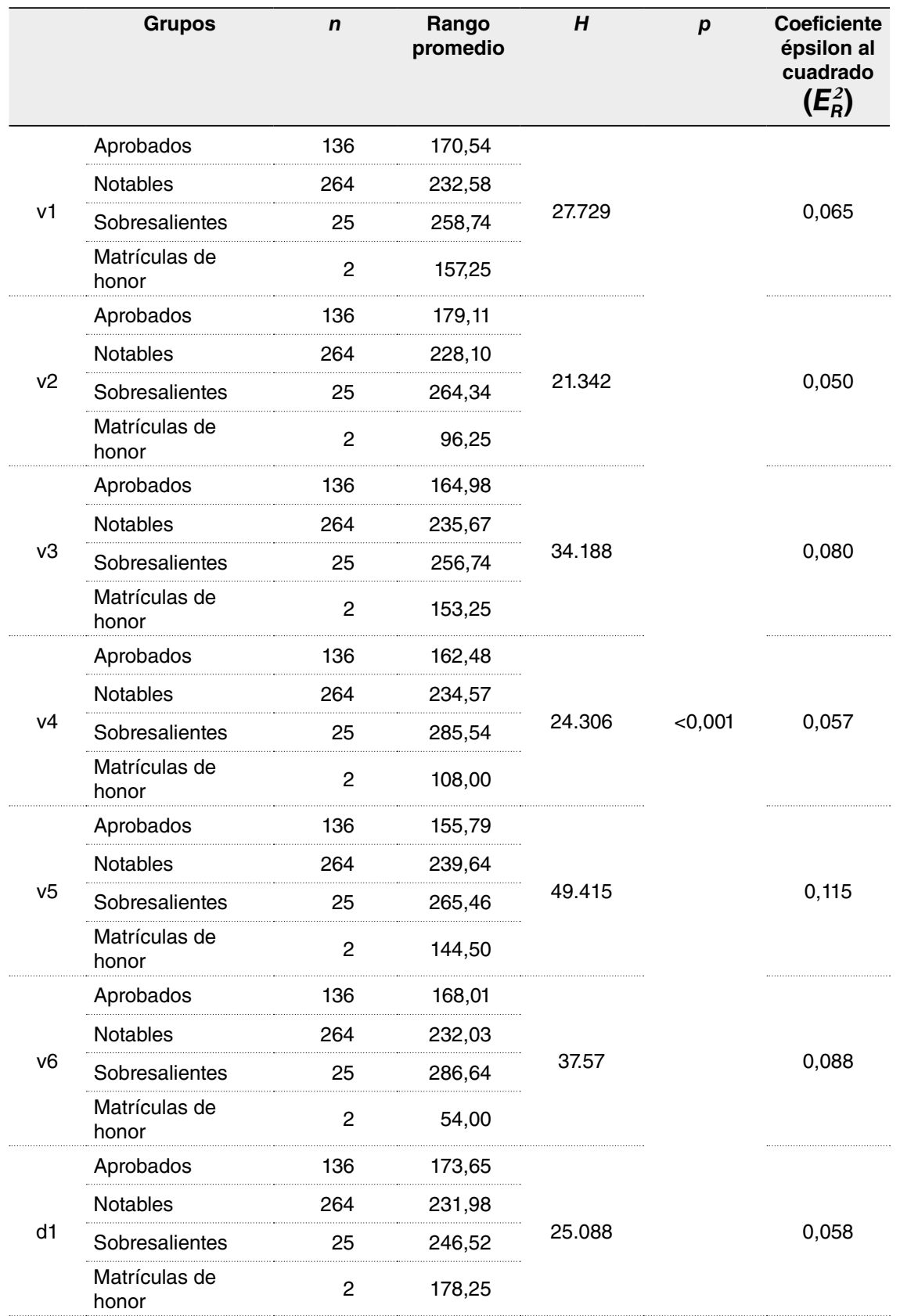




\begin{tabular}{|c|c|c|c|c|c|c|}
\hline \multirow{4}{*}{$d 2$} & Aprobados & 136 & 175,62 & \multirow{4}{*}{21.583} & & \multirow{4}{*}{0,050} \\
\hline & Notables & 264 & 232,13 & & & \\
\hline & Sobresalientes & 25 & 232,58 & & & \\
\hline & $\begin{array}{l}\text { Matrículas de } \\
\text { honor }\end{array}$ & 2 & 198,25 & & & \\
\hline \multirow{4}{*}{ d3 } & Aprobados & 136 & 167,53 & \multirow{4}{*}{31.237} & & \multirow{4}{*}{0,073} \\
\hline & Notables & 264 & 235,28 & & & \\
\hline & Sobresalientes & 25 & 247,30 & & & \\
\hline & $\begin{array}{l}\text { Matrículas de } \\
\text { honor }\end{array}$ & 2 & 149,50 & & & \\
\hline \multirow{4}{*}{ d4 } & Aprobados & 136 & 182,83 & \multirow{4}{*}{16.682} & & \multirow{4}{*}{0,039} \\
\hline & Notables & 264 & 228,33 & & & \\
\hline & Sobresalientes & 25 & 234,24 & & & \\
\hline & $\begin{array}{l}\text { Matrículas de } \\
\text { honor }\end{array}$ & 2 & 188,25 & & & \\
\hline \multirow{4}{*}{ d5 } & Aprobados & 136 & 183,55 & \multirow{4}{*}{19.878} & & \multirow{4}{*}{0,046} \\
\hline & Notables & 264 & 224,92 & & & \\
\hline & Sobresalientes & 25 & 273,14 & & & \\
\hline & $\begin{array}{l}\text { Matrículas de } \\
\text { honor }\end{array}$ & 2 & 103,75 & & - $\cap \cap \cap 1$ & \\
\hline \multirow{4}{*}{ a1 } & Aprobados & 136 & 173,65 & \multirow{4}{*}{24.055} & 年, & \multirow{4}{*}{0,056} \\
\hline & Notables & 264 & 230,83 & & & \\
\hline & Sobresalientes & 25 & 246,12 & & & \\
\hline & $\begin{array}{l}\text { Matrículas de } \\
\text { honor }\end{array}$ & 2 & 335,25 & & & \\
\hline \multirow{4}{*}{ a2 } & Aprobados & 136 & 174,34 & \multirow{4}{*}{25.447} & & \multirow{4}{*}{0,059} \\
\hline & Notables & 264 & 230,80 & & & \\
\hline & Sobresalientes & 25 & 261,84 & & & \\
\hline & $\begin{array}{l}\text { Matrículas de } \\
\text { honor }\end{array}$ & 2 & 95,50 & & & \\
\hline \multirow{4}{*}{ a3 } & Aprobados & 136 & 166,74 & \multirow{4}{*}{34.194} & & \multirow{4}{*}{0,080} \\
\hline & Notables & 264 & 234,91 & & & \\
\hline & Sobresalientes & 25 & 259,96 & & & \\
\hline & $\begin{array}{l}\text { Matrículas de } \\
\text { honor }\end{array}$ & 2 & 93,75 & & & \\
\hline \multirow{4}{*}{ a4 } & Aprobados & 136 & 152,52 & \multirow{4}{*}{53.205} & & \multirow{4}{*}{0,124} \\
\hline & Notables & 264 & 241,80 & & & \\
\hline & Sobresalientes & 25 & 252,42 & & & \\
\hline & $\begin{array}{l}\text { Matrículas de } \\
\text { honor }\end{array}$ & 2 & 244,50 & & & \\
\hline
\end{tabular}

(Continúa en la página siguiente) 


\begin{tabular}{|c|c|c|c|c|c|c|}
\hline \multirow{4}{*}{ a5 } & Aprobados & 136 & 168,43 & \multirow{4}{*}{31.608} & \multirow{8}{*}{$<0,001$} & \multirow{4}{*}{0,074} \\
\hline & Notables & 264 & 232,57 & & & \\
\hline & Sobresalientes & 25 & 271,20 & & & \\
\hline & $\begin{array}{l}\text { Matrículas de } \\
\text { honor }\end{array}$ & 2 & 146,50 & & & \\
\hline \multirow{4}{*}{ a6 } & Aprobados & 136 & 164,29 & \multirow{4}{*}{38.719} & & \multirow{4}{*}{0,090} \\
\hline & Notables & 264 & 233,80 & & & \\
\hline & Sobresalientes & 25 & 282,62 & & & \\
\hline & $\begin{array}{l}\text { Matrículas de } \\
\text { honor }\end{array}$ & 2 & 122,25 & & & \\
\hline
\end{tabular}

Fuente: elaboración propia.

de autoeficacia académica se esforzó por llevar a cabo otras actividades complementarias relacionadas con los estudios que realizaban $(H=31.237 ; p>0,001$; $\left.E_{R}^{2}=0,073\right)$. Finalmente, las observaciones llevadas a cabo en torno a la variable absorción sirvieron para definir un perfil de alumno caracterizado por sentirse feliz cuando realizaba tareas de clase $\left(H=34.194 ; p>0,001 ; E_{R}^{2}=0,080\right)$, por estar implicado en sus estudios $\left(H=53.205 ; p>0,001 ; E_{R}^{2}=0,124\right)$, por perder la noción del tiempo mientras llevaba a cabo actividades de clase $\left(H=31.608 ; p>0,001 ; E_{R}^{2}=0,074\right)$ y por no tener dificultades para hacer las tareas relacionadas con su formación $\left(H=38.719 ; p>0,001 ; E_{R}^{2}=0,090\right)$.

\section{Conclusiones}

En relación con la hipótesis planteada en esta investigación y teniendo en cuenta los resultados obtenidos, se concluye que los participantes que alcanzaron puntuaciones altas en la escala de compromiso mostraron al mismo tiempo creencias positivas respecto a su autoeficacia académica y a sus buenas expectativas de rendimiento. Las diferencias que se encontraron en cuanto a las calificaciones que esperaban lograr (aprobado, notable, sobresaliente y matrícula) respecto a las variables de compromiso analizadas (vigor, dedicación y absorción) fueron significativas. Así, aquellos estudiantes de grado que pensaban alcanzar buenos resultados académicos obtuvieron al mismo tiempo puntuaciones altas en la escala de compromiso. Por tanto, los que tenían un compromiso alto tenían creencias de autoeficacia académica también altas.

Estos resultados apuntan en la dirección de los obtenidos por Salanova et al. (2005) y Bresó, Salanova y Schaufeli (2007), que confirman la relación entre el compromiso y la autoeficacia académica. Los estudiantes implicados en su proceso formativo piensan que van a obtener buenos resultados, lo que los predispone al compromiso con las tareas y al esfuerzo en el logro de los objetivos académicos. En este caso, los universitarios con creencias de autoeficacia académica alta eran aquellos que realizaban las tareas de clase con energía, se levantaban con ganas de ir a la facultad, eran persistentes en el logro de sus objetivos 
pese a las dificultades, hacían actividades complementarias y se sentían felices realizando las tareas encomendadas. Compartimos con Liébana-Presa et al. (2018) que, a mayor autoeficacia académica, menor agotamiento y frustración y mejores puntuaciones en las distintas dimensiones del compromiso académico.

Estas conductas identificadas en los participantes del estudio coinciden con las que han señalado otros autores (Jang, Reeve y Deci, 2010; Cavazos y Encinas, 2016), al definir el compromiso académico como un estado mental positivo en relación con el trabajo y las tareas formativas. Reflejan una alta implicación, participación activa, intensidad y emoción, dedicación y persistencia para llevar a cabo las actividades de aprendizaje y lograr metas que cada uno se propone. Por tanto, hablamos de alumnos con niveles elevados de compromiso académico, que se perciben más eficaces en sus estudios, lo que puede traducirse en un mayor éxito en la formación en el presente y en el futuro, pero no solo en el plano académico, sino también en el profesional. Por eso resulta relevante el análisis del compromiso curricular, porque desde una perspectiva preventiva del fracaso y del abandono universitario ayuda a lograr una buena adaptación a los estudios y a conseguir la graduación que le permitirá en el futuro acceder al mundo laboral. Si los estudiantes reciben una buena orientación que les ayude a valorar la importancia que tiene la formación, a clarificar sus intereses, a definir un proyecto académico realista y a comprometerse con el logro de los objetivos que cada uno se proponga, tendrá más posibilidades de que su trayectoria académico-profesional sea más favorable y exitosa.

Aunque los resultados de la investigación son interesantes, sería necesario contrastarlos con otras muestras de alumnos de diferentes ramas de conocimiento, para valorar si también es significativa la incidencia del compromiso académico en las expectativas de rendimiento. Como señalan Liébana-Presa, Fernández-Martínez y Morán-Astorga (2017), es posible que se puedan producir diferencias en los resultados en función de las distintas titulaciones de los universitarios. Asimismo, sería conveniente profundizar en esta relación de variables a través de análisis de corte cualitativo, que permitieran ahondar en las creencias y en las autopercepciones que tienen los estudiantes respecto a su trayectoria formativa y a las expectativas de éxito. Finalmente, cabe destacar que los exámenes estadísticos ensayados en este trabajo apuntan a la posible relación existente entre el compromiso y las expectativas de autoeficacia académica. Esto lleva a la necesidad de realizar investigaciones más robustas que contribuyan a confirmar esta posible correspondencia entre dichas variables.

En cualquier caso, los hallazgos alcanzados en el presente trabajo tienen implicaciones directas en el proceso formativo del alumnado. Así, sería necesario y conveniente diseñar propuestas educativas y orientadoras en las que se trabajara la importancia del compromiso con la formación recibida como una variable que podría determinar la permanencia y el éxito académico, así como la mejora del proceso de aprendizaje de los estudiantes. Desde esta perspectiva, el diseño de acciones formativas de carácter preventivo en las que se abordara la implicación académica podría contribuir a reducir las elevadas tasas de fracaso, prolongación y abandono de la universidad. 


\section{Referencias bibliográficas}

Álvarez-Pérez, P.R.; López-Aguilar, D. y Valladares-Hernández, R.A. (2021). La influencia del engagement en las trayectorias formativas de los estudiantes de bachillerato. Estudios Sobre Educación (ESE), 40, 27-50. $<$ https://doi.org/10.15581/004.40.27-50>

Arriaga, J.; Burillo, V.; Carpeñol, A. y Casaravilla, A. (2011). Caracterización de los tipos de abandono: Dividamos el problema y venceremos más fácilmente. I Conferencia Latinoamericana sobre el Abandono en la Enseñanza Superior. Managua: Universidad Nacional Autónoma de Nicaragua.

Bandura, A. (1982). Self-efficacy mechanism in human agency. American Psychologist, 2, 122-147. <https://doi.org/10.1037/0003-066X.37.2.122>

Belhumeur, S.; Barrientos, A. y Renata-Salazar, A. P. (2016). Niveles de estrés de la población estudiantil en Costa Rica: Diferencias en función de las variables nivel socioeconómico, rendimiento académico, nivel académico y zona geográfica. Psychology, Society, \& Education, 8(1), 13-22. $<$ https://doi.org/10.25115/psye.v8i1.457>

Bernardo, A.; Cerezo, R.; Rodríguez, L. y Tuero, E. (2015). Predicción del abandono universitario: Variables explicativas y medidas de prevención. Fuentes: Revista de la Facultad de Ciencias de la Educación, 16, 63-84. <https://doi.org/10.12795/revistafuentes.2015.i16.03>

Bethencourt, J.T.; Cabrera, L.; Hernández, J.; Álvarez, P. y González, M. (2008). Variables psicológicas y educativas en el abandono universitario. Revista Electrónica de Investigación Psicoeducativa, 18, 603-622. <http://dx.doi.org/10.25115/ejrep.v6i16.1298>

Bresó, E. y Gracia, E. (2007). Bienestar psicológico en profesionales y estudiantes universitarios: Un estudio comparativo desde la Psicología Organizacional Positiva. Revista de Psicología Social Aplicada, 17, 23-38. Recuperado de <https://dialnet. unirioja.es/servlet/articulo?codigo=2934547>.

Bresó, E.; Salanova, M. y Schaufeli, W.B. (2007). In Search of the "Third Dimension" of burnout: Efficacy or inefficacy. Applied Psychology: An International Rewiev, 56(3), 460-478. <https://doi.org/10.1111/j.1464-0597.2007.00290.x>

Carmona-Halty, M.; Schaufeli, W. y Salanova, M. (2017). The Utrecht Work Engagement Scale for Students (UWES-9S): Factorial Validity, Reliability, and Measurement Invariance in a Chilean Sample of Undergraduate University Students. Frontiers in Psychology, 10, 1-5. $<$ https://doi.org/10.3389/fpsyg.2019.01017>

Casas, Y. y Blanco, A. (2017). Testing Social Cognitive Career Theory in Colombian Adolescent Secondary Students: A study in the field of mathematics and science. Revista Complutense de Educación, 28(4), 1173-1192. <https://doi.org/10.5209/RCED.52572>

Cavazos, J. y Encinas, F.C. (2016). Influencia del engagement académico en la lealtad de estudiantes de posgrado: Un abordaje a través de un modelo de ecuaciones estructurales. Estudios Gerenciales, 32, 228-238. <https://doi.org/10.1016/j.estger.2016.07.001>

Chen, R. (2012). Institutional Characteristics and College Student Dropout Risks: A Multilevel Event History Analysis. Research in Higher Education, 53, 487-505. <https://doi.org/10.1007/s11162-011-9241-4> 
Сно, E. (2016). Making Reliability Reliable: A Systematic approach to reliability coefficients. Organizational Research Methods, 19, 651-682. $<$ https://doi.org/10.1177/1094428116656239>

Coates, H. y McCormick, A. (eds.) (2014). Engaging university students: International insights from system-wide studies. Dordrecht: Springer.

Faul, F.; Erdfelder, E.; Lang, A.G. y Buchner, A. (2007). G*Power 3: A flexible statistical power analysis program for the social, behavioral, and biomedical sciences. Behavior Research Methods, 39(2), 175-191. <https://doi.org/10.3758/BF03193146>

Figuera, P.; Torrado, M.; Dorio, I. y Freixa, M. (2015). Trayectorias de persistencia y abandono de estudiantes universitarios no convencionales: Implicaciones para la Orientación. Revista Electrónica Interuniversitaria de Formación del Profesorado, 18(2), 107-123. $<$ https://doi.org/10.6018/reifop.18.2.220101>

George, D. y Mallery, M. (2001). Using SPSS for Windows step by step: A simple guide and reference. Boston, MA: Alyin y Bacon.

GiL-Monte, P.R. (2003). El síndrome de quemarse por el trabajo (síndrome de burnout) en profesionales de enfermería. Revista Eletrônica InterAção Psy, 1, 19-33. Recuperado de $<$ https://cutt.ly/9bDbLUN $>$.

Holgado, F.; SuÁrez, J.C. y Morata, M.D.L.A. (2019). Modelos de Ecuaciones Estructurales, desde el «Path Analysis» al Análisis Multigrupo: Una Guía Práctica con LISREL. Madrid: Sanz y Torres.

Íñiguez, T.; Elboj, C. y Valero, D. (2016). La Universidad del Espacio Europeo de Educación Superior ante el abandono de los estudios de grado: Causas y propuestas estratégicas de prevención. Educar, 52(2), 285-313. $<$ https://doi.org/10.5565/rev/educar.674>

Jang, H.; Reeve, J. y Deci, E.L. (2010). Engaging students in learning activities: It is not autonomy support or structure but autonomy support and structure. Journal of Educational Psychology, 102(3), 588-600. <https://doi.org/10.1037/a0019682>

Jurado, P.; Figuera, P. y Llanes, J. (2019). Análisis de las motivaciones y de las expectativas de los estudiantes de máster en Educación. Educar, 55(2), 325-341. $<$ https://doi.org/10.5565/rev/educar.1016>

Lent, R.W.; Taveira, M.; Figuera, P.; Dorio, I.; Faria, S. y Gonçalves, A.M. (2017). Test of the social cognitive model of well-being in Spanish college students. Journal of Career Assessment, 25(1), 135-143. $<$ https://doi.org/10.1177/1069072716657821>

Liébana-Presa, C.; Fernández-Martínez, C. y Morán-Astorga, C. (2017). Relación entre la inteligencia emocional y el burnout en estudiantes de enfermería. Psychology, Society, \& Education, 9(3), 335-345. $<$ https://doi.org/10.25115/psye.v9i3.856>

Liébana-Presa, C.; Fernández-Martínez, M.E.; VÁzquez Casares, A.M.; LópezAlonso, A.I. y Rodríguez-Borrego, M.A. (2018). Burnout y engagement en estudiantes universitarios de enfermería. Enfermería Global, 17(2), 131-152. $<$ https://doi.org/10.6018/eglobal.17.2.268831>

Martínez, I.M. y Salanova, M. (2003). Niveles de burnout y engagement en estudiantes universitarios: Relación con el desempeño y desarrollo profesional. Revista de Educación, 330, 361-384. Recuperado de <https://www.educacionyfp.gob. es/dam/jcr:185fe08c-621e-4376-b0e7-22d89b85e786/re3301911213-pdf.pdf>. 
Martos, A.; Pérez, M.C.; Molero, M.M.; Gázquez, J.J.; Simón, M.M. y BarraGán, A.B. (2018). Burnout y engagement en estudiantes de Ciencias de la Salud. European Journal of Investigation in Health, Psychology and Education, 8(1), 23-36. <https://doi.org/10.30552/ejihpe.v8i1.223>

Muñoz, J.A. y Amón, I. (2013). Técnicas para detección de outliers multivariantes. Revista en Telecomunicaciones e Informática, 3(5), 11-25. Recuperado de <http:// hdl.handle.net/20.500.11912/6582>.

Parra, P. (2010). Relación entre el nivel de Engagement y el rendimiento académico teórico/práctico. Revista de Educación en Ciencias de la Salud, 7(1), 57-63. Recuperado de <http://www2.udec.cl/ofem/recs/anteriores/vol712010/revbi b71a.pdf>.

Raykov, T. y Marcoulides, G.A. (2017). Thanks Coefficient Alpha, We Still Need You! Educational and Psychological Measurement, 79(1), 200-210. <https://doi.org/10.1177/0013164417725127>

RuÉ, J. (2007). Enseñar en la universidad: El EEES como reto para la educación superior. Madrid: Narcea.

Salanova, M.; Martínez, I.M.; Bresó, E.; Llorens, S. y Grau, R. (2005). Bienestar psicológico en estudiantes universitarios: Facilitadores y obstaculizadores del desempeño académico. Anales de Psicología, 21(1), 170-180. Recuperado de <https://www.redalyc.org/pdf/167/16721116.pdf>.

Savage, M.W.; Strom, R.E.; Ebesu Hubbard, A.S. y Aune, K.S. (2017). Commitment in College Student Persistence. Journal of College Student Retention: Research, Theory \& Practice, 21(2), 242-264. <https://doi.org/10.1177/1521025117699621>

Schaufeli, W.B. y Bakker, A.B. (2003). UWES: Utrecht Work Engagement Scale. Utrecht: Utrecht University.

Schaufeli, W.B.; Martínez, I.M.; Pinto, A.M.; Salanova, M. y Bakker, A.B. (2002a). Burnout and engagement in university students: A cross-national study. Journal of Cross-Cultural Psychology, 33(5), 464-481. <https://doi.org/10.1177/0022022102033005003>

Schauffeli, W.B. y Salanova, M. (2007). Efficacy or inefficacy, that's the question: Burnout and work engagement, and their relationships with efficacy believes. Anxiety, Stress and Coping, 20, 177-196. <https://doi.org/10.1080/10615800701217878>

Schaufeli, W.B.; Salanova, M.; González-Romá, V. y Bakker, A. (2002b). The measurement of burnout and engagement: A confirmatory factor analytic approach. Journal of Happiness Studies, 3, 71-92. <https://doi.org/10.1023/A:1015630930326>

Tinto, V. (1975). Dropout from Higher Education: A Theoretical Synthesis of Recent Research. Review of Educational Research, 43(1), 89-125. <https://doi.org/10.3102/00346543045001089>

- (2017). Through the Eyes of Students. Journal of College Student Retention: Research, Theory o Practice, 19(3), 254-269. <https://doi.org/10.1177/1521025115621917>

ToмсzaK, M. y ToмсzaK, E. (2014). The need to report effect size estimates revisited: An overview of some recommended measures of effect size. Trends Sport Sciences, 1(21), 19-25. Recuperado de <https://cutt.ly/lbDbRAI>. 
Torrado, M. y Figuera, P. (2019). Estudio longitudinal del proceso de abandono y reingreso de estudiantes de Ciencias Sociales: El caso de Administración y Dirección de Empresas. Educar, 55(2), 401-417. <https://doi.org/10.5565/rev/educar.1022>

Ventura-León, J.L. y Caycho-Rodríguez, T. (2017). El coeficiente Omega: Un método alternativo para la estimación de la confiabilidad. Revista Latinoamericana de Ciencias Sociales, Niñez y Juventud, 15(1), 625-627. Recuperado de <https:// www.redalyc.org/pdf/773/77349627039.pdf>.

Wilkins, S.; Butt, M.M.; Kratochvil, D. y Balakrishnan, M.S. (2016). The Effects of Social Identification and Organizational Identification on Student Commitment, Achievement and Satisfaction in Higher Education. Studies in Higher Education, 41(12), 2232-2252.

$<$ https://doi.org/10.1080/03075079.2015.1034258> 